\title{
Avaliação da estabilidade e atividade antioxidante de uma emulsão base não-iônica contendo resveratrol
}

\author{
Marcela Kist Lange', Graziela Heberlée ${ }^{*}$, Denise Milão² \\ ${ }^{1}$ Faculdade de Farmácia, Centro de Ciências Biológicas e da Saúde, Universidade do Vale do Taquari-UNIVATES, \\ ${ }^{2}$ Faculdade de Farmácia, Departamento de Desenvolvimento e Produção de Medicamentos, \\ Pontifícia Universidade Católica do Rio Grande do Sul.
}

\begin{abstract}
Vários são os fatores que podem ocasionar a instabilidade de uma emulsão, destacando-se a oxidação, reação prevenida pelo emprego de antioxidantes. O butil-hidróxi-tolueno (BHT) tem sido um dos antioxidantes sintéticos mais utilizados em formulações cosméticas, porém, a busca da indústria farmacêutica e cosmética pelo emprego de produtos de origem natural tem sido cada vez maior. Visto isso, o objetivo do presente trabalho foi a incorporação do resveratrol, um composto fenólico encontrado principalmente em uvas bem como em vinhos tintos, em uma emulsão base não-iônica para avaliação do perfil de estabilidade e atividade antioxidante em comparação a uma emulsão base não-iônica contendo o BHT. O perfil de estabilidade foi analisado pela observação das características organolépticas, determinação do $\mathrm{pH}$ e espalhabilidade, e atividade antioxidante através do teste com o radical livre 2,2-difenil-1-picrilhidrazila (DPPH). Em relação à estabilidade, a altas temperaturas, a emulsão contendo BHT mostrou-se superior à emulsão contendo resveratrol. Pela análise da atividade antioxidante, o resveratrol tanto na sua forma de extrato seco, como quando incorporado na emulsão, demonstrou significativa superioridade em relação ao BHT, podendo ser sua utilização uma alternativa viável em preparações cosméticas, devido ao seu grande potencial antioxidante.
\end{abstract}

Unitermos: Emulsões/ estabilidade. Antioxidantes. Butil-hidróxi-tolueno. Resveratrol.

There are several factors that can lead to the instability of an emulsion, highlighting the oxidation, a reaction prevented by the use of antioxidants. The butylated hydroxytoluene (BHT) has been one of the most used synthetic antioxidants in cosmetic formulations; however, pharmaceutical and cosmetic industries have shown considerable interest regarding the search for the use of natural products. Based on this, the objective of this work was the incorporation of resveratrol, a natural phenolic compound found mainly in grapes as well as in red wines, into a non-ionic emulsion basis for assessing the profile of stability and antioxidant activity, as compared with a non-ionic basis emulsion containing BHT. The profile of stability was examined by the observation of the organoleptic characteristics, determination of $\mathrm{pH}$ and spreadability, and the antioxidant activity through the Radical Scavenging DPPH test. The results showed that the emulsion containing BHT was more stable than the emulsion containing resveratrol, when high temperature was used. For the analysis of the antioxidant activity, the resveratrol, in both forms of incorporation, showed significant antioxidant activity in comparison to BHT, suggesting that resveratrol may be a viable antioxidant alternative to be used into cosmetic preparations.

Uniterms: Emulsions/stability. Antioxidants. Butylated hydroxytoluene. Resveratrol.

\section{INTRODUÇÃO}

O desenvolvimento de novas formulações no mer-

\footnotetext{
*Correspondência: G. Heberlé. Curso de Farmácia - UNIVATES, Centro Universitário, Centro de Ciências Biológicas e da Saúde, Rua Avelino Tallini, 171- Bairro Universitário, 95900-000 - Lajeado - RS, Brasil. E-mail: gheberle@univates.br.
}

cado farmacêutico e cosmético tem se dado de maneira crescente nos últimos anos, sendo as emulsões amplamente utilizadas tanto para a incorporação de fármacos, como também de ativos cosméticos. Para a obtenção do sucesso terapêutico, a forma farmacêutica deve apresentar-se estável, o que torna a avaliação de sua estabilidade um fator fundamental. Esta pode ser avaliada através do teste de 
estabilidade acelerada, que tem o objetivo de verificar, em condições específicas e controladas, a capacidade de um produto manter as mesmas características e propriedades ao longo de sua vida útil (Alves et al.,1999; Zanin et al., 2001; Brasil, 2004; Zunino, 2007).

Um dos fatores que pode ocasionar a instabilidade de uma emulsão é a reação de oxidação, resultando em alterações no odor e aparência do produto, podendo ser causada pelo oxigênio atmosférico ou ainda pela ação de microorganismos, especialmente na fase oleosa. Essa causa de instabilidade pode ser evitada com a adição de antioxidantes na formulação, dentre os quais se destaca o BHT, um antioxidante sintético amplamente empregado nas formulações cosméticas. Porém, atualmente há uma busca notável pela utilização de produtos de origem natural, tanto pela indústria farmacêutica, como cosmética, visando atender às exigências do mercado (Silva, Soares, 1996).

Os compostos fenólicos, substâncias que apresentam pelo menos um anel aromático, onde ao menos um hidrogênio é substituído, são amplamente distribuídos no reino vegetal e apresentam destaque especial por exercer em frutas e verduras a ação antioxidante necessária para o funcionamento de células vegetais. O resveratrol é um composto fenólico, pertencente ao grupo dos flavonóides, encontrado em cerca de setenta espécies vegetais, em particular, presente abundantemente em uvas (Vitis vinifera e Vitis labrusca), tanto na forma cis (cis-3,5,4'-triidroxiestilbeno), como trans (trans-3,5,4'-triidroxiestilbeno), com sua síntese ocorrendo principalmente nas cascas dos frutos (Carvalho et al., 2003; Andrade, 2004; Avello, Suwalsky, 2006; Ramalho, Jorge, 2006).

Sendo assim, este trabalho visa a comparação da estabilidade e da atividade antioxidante de duas emulsões, contendo as mesmas matérias-primas (mesmo lote e fornecedor), porém, uma contendo o antioxidante BHT de concentração de uso já conhecida e outra contendo resveratrol na forma de extrato seco.

Neste artigo, serão apresentados os resultados da avaliação do comportamento de ambas as formulações, frente a condições específicas tanto no que diz respeito ao tempo, como em relação ao armazenamento. Serão apresentados também, resultados quanto à viabilidade de emprego do antioxidante resveratrol em formulações de uso tópico.

\section{MATERIAL E MÉTODOS}

\section{Insumos}

Crodabase CR2 ${ }^{\circledR}$ (Alpha Química - lote: 0393/07);
Polawax $^{\circledR}($ álcool cetoestearílico monoestearato de sorbitano polioxietileno 20 O.E) (Delaware - lote: 240107); metilparabeno (Delaware - lote: 060706); propilparabeno (Delaware - lote: 141106); propilenoglicol (Galena - lote: 0701008404); vaselina líquida (Synth - lote: 87590); BHT (Synth - lote: 79662); Resveratrol ${ }^{\circledR}$ - Extrato seco 8,19\% em resveratrol (Galena - lote: GK060926); água destilada; DPPH (2,2-difenil-1-picrilhidrazila); metanol (Nuclear lote: 06040521 ) e potes de propileno, parede dupla com tampa rosqueável e tampa interna de PVC.

\section{Preparação das emulsões base não-iônicas}

Para a preparação das emulsões bases não-iônicas, as matérias-primas foram separadas conforme a hidro ou lipofilia. Os componentes da fase aquosa foram dissolvidos na chapa de aquecimento, em béquer de $1000 \mathrm{~mL}$, à temperatura de $80^{\circ} \mathrm{C}$ e os componentes da fase oleosa foram fundidos em banho-maria, em béquer de $1000 \mathrm{~mL}$, a uma temperatura de até $75^{\circ} \mathrm{C}$. Depois de alcançada a temperatura de ambas fases oleosa e aquosa, procedeu-se a adição lenta da fase aquosa sobre a fase oleosa, sob agitação constante de $9000 \mathrm{rpm}$, velocidade 4, com misturador IKA RW 20n até o arrefecimento. Durante os primeiros vinte minutos utilizou-se o agitador naval e após o agitador âncora por doze minutos.

Na preparação de $1000 \mathrm{~g}$ da emulsão base não iônica contendo o BHT (EBHT), adicionou-se o mesmo juntamente com a fase oleosa da formulação. Já para a preparação da mesma quantidade da emulsão base não iônica contendo o extrato seco de resveratrol (ER), bem como para a emulsão base não iônica Polawax, também contendo o extrato seco de resveratrol como antioxidante (EPR), incorporou-se o mesmo na concentração de $2 \%$, previamente diluído em $10 \mathrm{~mL}$ de água destilada, após o arrefecimento da emulsão.

\section{Teste de estabilidade acelerada}

As emulsões, EBHT e ER foram armazenadas em triplicata à temperatura ambiente a $20^{\circ} \mathrm{C}\left(20,45 \pm 1,45^{\circ} \mathrm{C}\right)$, na estufa a $45^{\circ} \mathrm{C}\left(45,00 \pm 0,15^{\circ} \mathrm{C}\right)$ e na geladeira a $4{ }^{\circ} \mathrm{C}$ $\left(4,10 \pm 1,56^{\circ} \mathrm{C}\right)$, em todas as condições ao abrigo da luz. $\mathrm{O}$ estudo de estabilidade foi realizado durante dois meses, iniciando com a centrifugação das formulações. As determinações do $\mathrm{pH}$ e parâmetros organolépticos foram realizadas nos tempos $0,15,30,45$ e 60 dias, e as análises de espalhabilidade foram realizadas nos tempos 30 e 60 dias. O tempo zero foi considerado o momento em que se realizou o primeiro registro de um parâmetro analisado (Brasil, 2004). 


\section{Centrifugação}

As bases não iônicas EBHT, ER e EPR foram submetidas a centrifugação na centrífuga digital de tubos Q-222 D18/28 QUIMIS a $3000 \mathrm{rpm}, 25^{\circ} \mathrm{C}$ por 30 minutos após 24 horas da sua produção, com o objetivo de verificar uma possível instabilidade inicial (Brasil, 2004).

\section{Determinação das características organolépticas}

As características organolépticas avaliadas foram: aspecto, cor e odor. Para a avaliação, adicionou-se uma alíquota de cada amostra em vidro relógio, tendo sido as características analisadas sobre um fundo branco em comparação a padrões, sendo estes, três potes das bases não iônicas EBHT e ER, armazenados à temperatura de $19^{\circ} \mathrm{C}\left(18,78 \pm 0,76^{\circ} \mathrm{C}\right)$ e ao abrigo da luz. O aspecto e a cor foram avaliados visualmente e o odor diretamente através do olfato (Brasil, 2004; Spellmeier, 2005).

\section{Determinação do $\mathrm{pH}$}

Determinou-se o pH de cada formulação utilizandose uma solução a $10 \%(\mathrm{~m} / \mathrm{v})$, obtida pela dispersão de $2 \mathrm{~g}$ da formulação em água destilada com o auxílio do agitador magnético MA 085 na velocidade 4 por 15 minutos, para amostras armazenadas à temperatura ambiente, estufa e geladeira, sendo as determinações realizadas com pHmetro previamente calibrado com soluções-tampão pH 4 e pH 7. Os resultados correspondem à média de três determinações (Knorst, 1991; Archondo, 1999; Milão, 2001).

\section{Determinação da espalhabilidade}

Para determinação da espalhabilidade uma placamolde circular de vidro (diâmetro $=20 \mathrm{~cm}$; espessura $=$ $0,3 \mathrm{~cm}$ ), com orifício central de 1,2 cm de diâmetro, foi colocada sobre uma placa suporte de vidro $(20 \mathrm{~cm}$ x 20 $\mathrm{cm})$. Sob essa placa posicionou-se uma folha de papel milimetrado e uma fonte luminosa. A amostra foi introduzida no orifício da placa e a superfície foi nivelada com espátula. Após, a placa-molde foi cuidadosamente retirada. Sobre a amostra foi colocada uma placa de vidro de peso pré-determinado. Depois de um minuto, foi calculada a superfície abrangida, através da medição do diâmetro em duas posições opostas, com auxílio da escala do papel milimetrado e com posterior cálculo do diâmetro médio. Este procedimento foi repetido acrescentando-se novas placas, em intervalos de um minuto, registrando-se a cada determinação a superfície abrangida pela amostra e o peso da placa adicionada até um número máximo de 19 placas
(Knorst, 1991; Milão, 2001; Spellmeier, 2005).

A espalhabilidade (Ei), determinada a $24 \pm 2{ }^{\circ} \mathrm{C}$, foi calculada através da equação:

$\mathrm{Ei}=\frac{\mathrm{d}^{2} \mathrm{x} \pi}{4}$

Onde: $\mathrm{Ei}=$ espalhabilidade da amostra para peso $\mathrm{i}$ $\left(\mathrm{mm}^{2}\right) ; \mathrm{d}=$ diâmetro médio $(\mathrm{mm})$

A espalhabilidade máxima foi considerada como o ponto no qual a adição de peso não ocasionou alterações significativas nos valores da espalhabilidade, e o esforço limite corresponde ao peso que resulta na espalhabilidade máxima.

\section{Avaliação da atividade antioxidante}

A capacidade antioxidante de ambas as formulações, EBHT e ER, bem como a comparação entre o antioxidante BHT e resveratrol, foi determinada através do teste com o radical livre DPPH, através do seqüestro desses radicais livres (Elmastas et al., 2006).

Nesse método é preparada uma solução $0,00013 \mathrm{M}$ de DPPH em metanol. Para a preparação das amostras, é pesada a quantidade de $0,0625 \mathrm{~g}$ de cada uma, com adição de $25 \mathrm{~mL}$ de metanol, resultando em uma solução de cada amostra na concentração de $0,0025 \mathrm{~g} / \mathrm{mL}$. Em seguida, é preparada uma solução de concentração $0,00001 \mathrm{~g} / \mathrm{mL}$ de cada amostra, as quais são novamente diluídas conforme Figura 1.

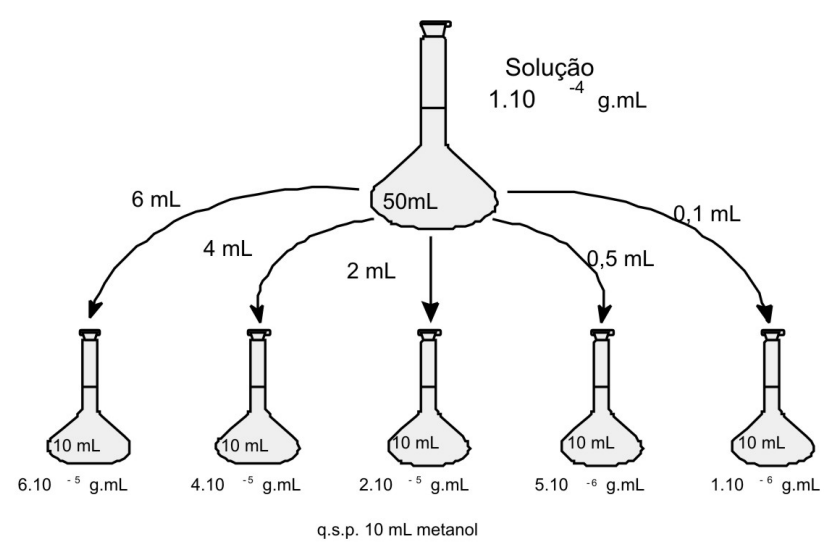

FIGURA 1 - Diluições realizadas para teste do radical DPPH.

Após a adição da solução de DPPH, aguardou-se 30 minutos para iniciar a leitura no espectrofotômetro no comprimento de onda $517 \mathrm{~nm}$. Além da solução com a amostra, foi realizada a leitura da solução controle contendo somente 1:3 DPPH e metanol.

Para avaliação da atividade captadora de radical 
livre foi calculada a porcentagem de inibição do DPPH pela equação:

\% inibição do DPPH $=\left[\left(\mathrm{A}_{0}-\mathrm{A}_{1}\right) / \mathrm{A}_{0} \times 100\right]$

Onde: $\mathbf{A}_{\mathbf{0}}=$ absorbância do controle; $\mathbf{A}_{\mathbf{1}}=$ absorbância da amostra.

\section{Análise estatística}

Para análise dos resultados do $\mathrm{pH}$, avaliação da espalhabilidade e atividade antioxidante, utilizou-se o programa SAE - Sistema de Apoio Estatístico (Ahlert, 2005), através dos testes estatísticos ANOVA, Teste t e Tukey, com níveis de confiança de $95 \%$.

\section{RESULTADOS E DISCUSSÃO}

\section{Teste de centrifugação}

A centrifugação foi realizada como análise preliminar, a fim de determinar qualquer sinal de instabilidade indicativa de necessidade de reformulação (Brasil, 2004). Após este teste, nenhuma das formulações, EBHT, ER e EPR, apresentaram alteração em relação ao seu aspecto original.

\section{Determinações organolépticas}

As formulações EBHT e ER foram analisadas frente a padrões, sendo eles três potes das bases não iônicas EBHT e ER, armazenados à temperatura de $19^{\circ} \mathrm{C}(18,78$ $\pm 0,76^{\circ} \mathrm{C}$ ), ao abrigo da luz.

À temperatura de $20{ }^{\circ} \mathrm{C}$, as formulações EBHT e ER permaneceram inalteradas durante todo o período de estudo de estabilidade (60 dias). A emulsão EBHT, armazenada à $45^{\circ} \mathrm{C}$, apresentou alterações somente após 45 dias, período após o qual se observou a presença de gotículas na superfície, indicando o início da separação de fases. Entretanto, em relação à cor e ao odor permaneceu inalterada. A emulsão ER apresentou-se com nítida separação de fases e odor alterado após 15 dias armazenada à $45^{\circ} \mathrm{C}$, mostrando-se instável quando submetida a essa temperatura, sendo descartadas para as próximas análises as demais amostras ER armazenadas nesta condição.

Para verificar se a instabilidade da emulsão ER, demonstrada pela nítida separação de fases, manter-se-ia mesmo com a alteração da base, preparou-se uma nova formulação utilizando-se a base auto-emulsionante não iônica Polawax (álcool cetoestearílico e monoestearato de sorbitano polioxietileno $20 \mathrm{OE}$ ). Essa nova formulação (EPR), sem adição do antioxidante BHT e contendo
$2 \%$ de extrato seco de resveratrol, foi armazenada nas mesmas condições da EBHT e ER, para comparação em relação à emulsão ER. Aos 15 dias, a formulação EPR, armazenada à $45^{\circ} \mathrm{C}$, apresentou nítida separação de fases, indicando que a instabilidade das emulsões contendo resveratrol quando submetidas ao calor não está relacionada necessariamente ao tipo de base utilizada, podendo esta ocorrer devido à evaporação de água da formulação e/ou pela ação do calor que acelera reações entre os componentes da mesma e ainda pelo extrato seco de resveratrol incorporado.

\section{Determinação do pH das formulações}

Pela realização deste teste, observa-se que em relação às diferentes condições de armazenamento, as amostras EBHT e ER apresentaram diferenças significativas (nível de confiança 95\%) quando comparadas uma com a outra. Cabe ressaltar que essa diferença já ocorre no tempo 0 , ou seja, as formulações têm originalmente valores de $\mathrm{pH}$ diferentes, apresentando-se a formulação EBHT com maior valor em relação à ER, como pode ser observado nas Figuras 2 e 3.

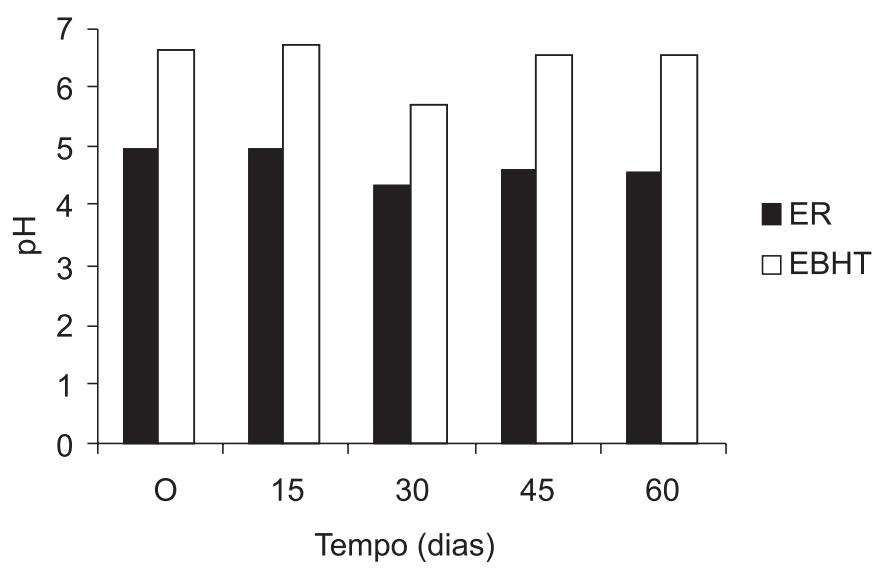

FIGURA 2 - Valores de $\mathrm{pH}$ da EBHT e ER armazenadas à $20^{\circ}$ $\mathrm{C}$ em função do tempo de armazenamento $(\mathrm{n}=3)$.

A comparação dos valores de $\mathrm{pH}$ da EBHT em relação à ER, ambas armazenadas a $45^{\circ} \mathrm{C}$, não foi realizada devido à instabilidade da ER quando submetida a esta condição; o que levou à eliminação da amostra. Entretanto, o pH manteve-se estável para cada formulação, não diferindo significativamente em relação ao tempo transcorrido e nas temperaturas de 4 e $20^{\circ} \mathrm{C}$.

\section{Avaliação da espalhabilidade}

Pela análise da espalhabilidade, foi possível obser- 


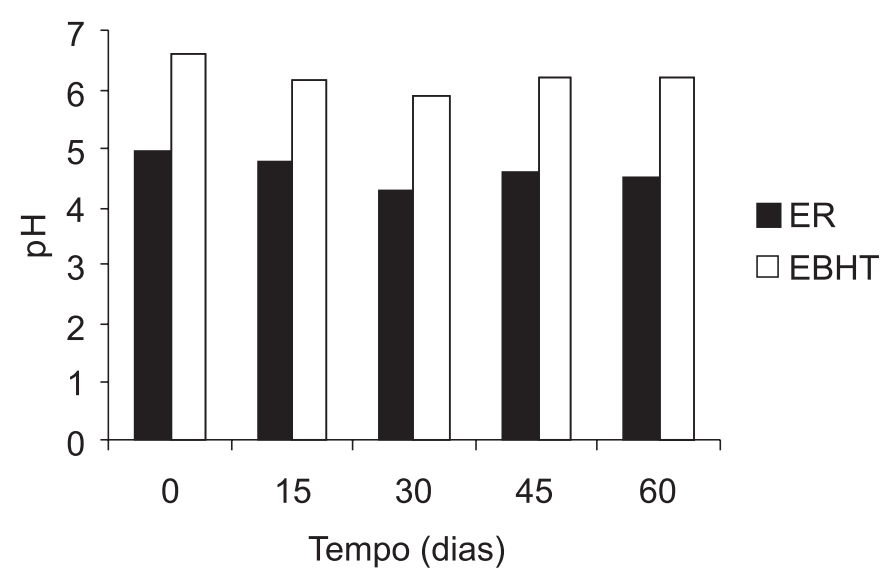

FIGURA 3 - Valores de $\mathrm{pH}$ da EBHT e ER armazenadas a $4^{\circ} \mathrm{C}$ em função do tempo de armazenamento $(n=3)$.

var que existe diferença significativa entre as formulações EBHT e ER.

Frente às diferentes condições de armazenamento, no período de 60 dias, constatou-se que para a EBHT, as formulações armazenadas a 20 e $4{ }^{\circ} \mathrm{C}$, apresentaram comportamentos semelhantes quanto à espalhabilidade, diferindo de maneira significativa em relação a ambas, a amostra submetida a $45^{\circ} \mathrm{C}$, como pode ser observado na Figura 4.

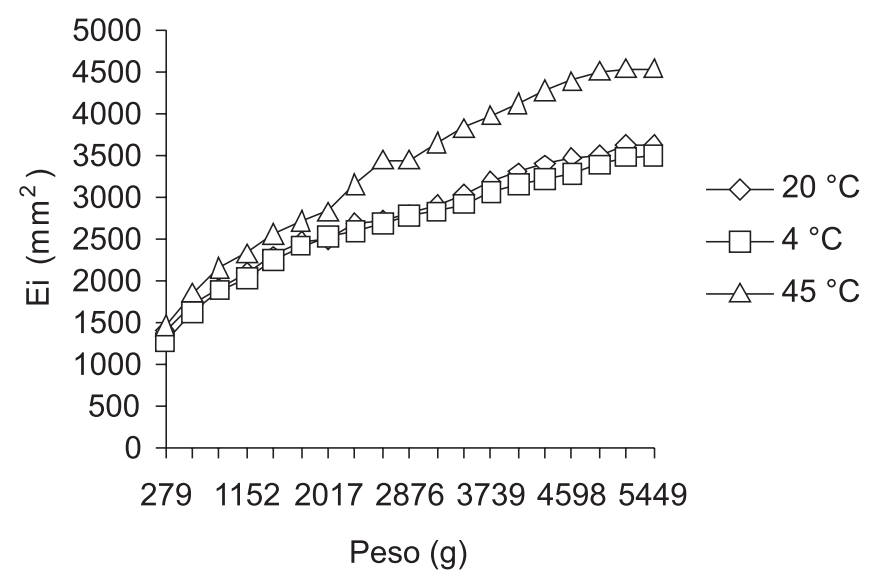

FIGURA 4 - Espalhabilidade (Ei) da EBHT (60 dias) nas diferentes condições de armazenamento em relação ao peso adicionado $(\mathrm{n}=3)$.

Já para a formulação ER armazenada a 20 e $4{ }^{\circ} \mathrm{C}$ não houve variação significativa nesses valores, ao longo dos 60 dias de análise (Figura 5). Por ter apresentado separação de fases não foi possível a análise da mesma a $45^{\circ} \mathrm{C}$.

Em relação ao tempo de armazenamento, nas diferentes condições, a EBHT alterou de maneira significativa o valor de sua espalhabilidade quando armazenada a $20 \mathrm{e}$ $4{ }^{\circ} \mathrm{C}$, porém não houve diferença significativa na amostra

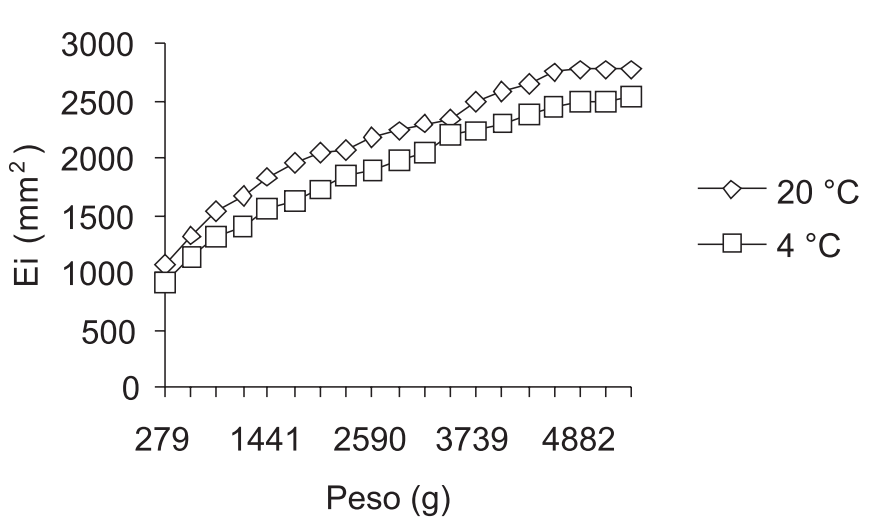

FIGURA 5 - Espalhabilidade (Ei) da ER (60 dias) nas diferentes condições de armazenamento em relação ao peso adicionado $(\mathrm{n}=3)$.

submetida a $45^{\circ} \mathrm{C}$ no decorrer dos 60 dias. Já a ER diferiu de maneira significativa sua espalhabilidade quando armazenada a $20^{\circ} \mathrm{C}$, porém essa diferença não foi observada na amostra submetida a $4{ }^{\circ} \mathrm{C}$ durante os 30 e 60 dias. Sendo assim, pode-se dizer que, de modo geral, a EBHT apresentou ao longo do período de análise, maior espalhabilidade que a ER, fato que pode ser explicado pela presença do extrato seco de resveratrol, um componente pulvurulento, que aumenta a consistência da formulação, diminuindo conseqüentemente a sua espalhabilidade.

\section{Avaliação da atividade antioxidante}

Para a avaliação da atividade antioxidante desempenhada pelas diferentes formulações EBHT e ER, bem como para comparação entre o BHT e o extrato seco de resveratrol, as amostras foram submetidas ao teste do radical DPPH.

Através da avaliação da atividade antioxidante do BHT em relação ao extrato seco de resveratrol, foi possível observar de maneira significativa a superioridade do resveratrol, quando avaliado tanto na sua forma de extrato seco como quando incorporado à emulsão (Figuras 6 e 7).

Esse resultado pode ser explicado ao observar-se a estrutura química da molécula do resveratrol. Esta apresenta dois anéis aromáticos e ligações duplas, formando intermediários relativamente estáveis, devido à ressonância do anel presente em sua estrutura, enquanto o BHT apresenta em sua estrutura apenas um anel aromático.

A avaliação da atividade antioxidante foi testada também entre as formulações EBHT e ER, contendo ambas o respectivo antioxidante na concentração de $0,05 \%$, e tendo sido armazenadas à temperatura de $20^{\circ} \mathrm{C}$. Nesta condição, observou-se novamente que há diferença significativa entre a utilização do extrato seco de resveratrol em relação ao BHT, sendo a formulação contendo resveratrol 


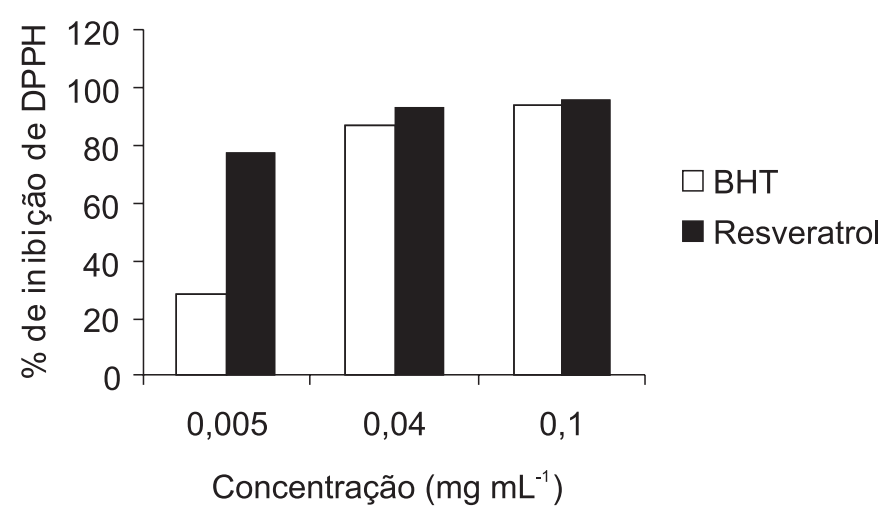

FIGURA 6 - Percentual de inibição de DPPH do BHT e do resveratrol $(n=3)$. Amostras de BHT e resveratrol preparadas conforme descrito na metodologia.

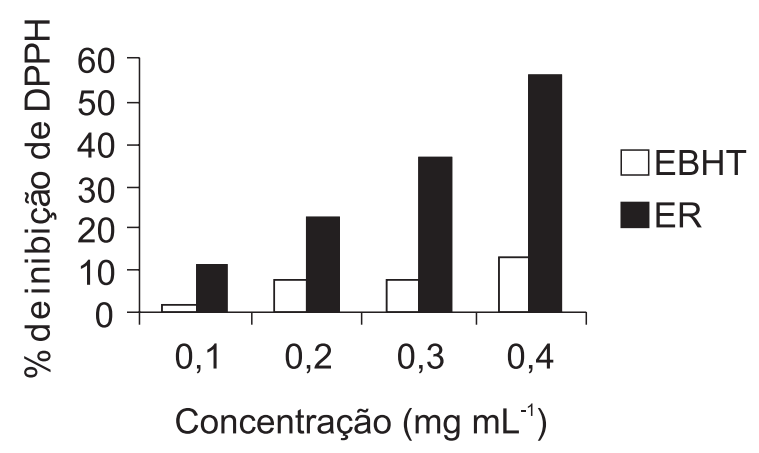

FIGURA 7 - Percentual de inibição de DPPH na EBHT e ER armazenadas à $20^{\circ} \mathrm{C}(\mathrm{n}=3)$.

superior em todas as concentrações testadas, mostrando sua atividade antioxidante, mesmo quando incorporado em uma emulsão (Figura 7).

$\mathrm{O}$ aumento da temperatura pode acelerar reações químicas nas formulações, ou ainda, neste caso, ocasionar perda de atividade antioxidante nas emulsões submetidas ao calor, armazenadas a $45^{\circ} \mathrm{C}$ durante o período de estudo. Devido ao fato da formulação ER apresentar-se instável já aos 15 dias, quando armazenada a $45^{\circ} \mathrm{C}$, realizou-se o teste de atividade antioxidante para verificar se o aumento de temperatura influi na sua atividade, comparando o percentual de inibição de DPPH apresentado pelas formulação ER armazenadas a 20 e $45^{\circ} \mathrm{C}$. Na maioria das concentrações, foram observadas diferenças significativas entre as formulações, sendo que a ER submetida ao calor apresentou diminuição do percentual de inibição de DPPH.

\section{CONCLUSÕES}

Em relação à análise preliminar da estabilidade da formulação contendo BHT e daquela contendo resveratrol, através da centrifugação das formulações, ambas apresen- taram-se estáveis, não sendo necessária a reformulação para a continuidade do estudo.

Os resultados obtidos neste trabalho demonstraram que a EBHT apresenta estabilidade superior em relação à ER, quando ambas são submetidas a altas temperaturas $\left(45^{\circ} \mathrm{C}\right)$.

$\mathrm{Na}$ avaliação da espalhabilidade de ambas as formulações, nas diversas condições de armazenamento, no decorrer dos 60 dias, a EBHT apresentou maiores valores de espalhabilidade. Cabe ressaltar que a espalhabilidade foi originalmente superior, o que já era esperado, já que a ER apresentava maior teor de sólidos na sua formulação, devido à incorporação do extrato seco contendo resveratrol.

$\mathrm{Na}$ avaliação da atividade antioxidante, o emprego do resveratrol justifica-se por ter sido observada sua diferenciada ação na formulação ER em relação à EBHT, que, mesmo após perda de parte de seu potencial antioxidante, quando submetida ao calor, mostrou-se superior ao BHT.

Sendo assim, o desenvolvimento de formulações cosméticas contendo resveratrol como antioxidante mostra-se uma alternativa viável, devido a sua notável superioridade em relação ao antioxidante sintético BHT. Entretanto, é mais aconselhável a utilização de extratos com maiores teores de resveratrol ou da molécula de forma isolada, para que sejam minimizados os efeitos de outras substâncias que constituem os extratos, como por exemplo, excipientes, nas características físico-químicas, bem como na estabilidade da formulação cosmética.

\section{REFERÊNCIAS}

AHLERT, L. SAE - Sistema de Apoio Estatístico. Lajeado, 2005. 1 CD-ROM.

ALVES, M. P.; FUNCK, J. A. B.; BITTENCOURT, C. F.; SILVA, M. A. S. Desenvolvimento e avaliação da estabilidade de bases dermatológicas não iônicas para incorporação de fármacos. Rev. Pharm. Tech. (Ed. port.), v.3, n.2, p.40-45, 1999.

ANDRADE, S. J. Propriedades farmacológicas e químicas do nutracêutico resveratrol. Santa Cruz do Sul, 2004. 79 f. [Monografia de Graduação. Curso de Farmácia. Universidade de Santa Cruz do Sul].

AVELLO, M.; SUWALSKY, M. Radicales libres, antioxidantes naturales y mecanismos de protección. Atenea (Concepc.). [online]. 2006, no 494, p.161-172. Disponível em: <http:// www.scielo.cl/scielo.php?script=sci_arttext\&pid=S071804 $622006000200010 \& \operatorname{lng}=\mathrm{es} \& \mathrm{nrm}=\mathrm{iso}>$. ISSN 0718-0462. Acesso em: 24 Out. 2007. 
BRASIL. Guia de Estabilidade de Produtos Cosméticos. Anvisa. Brasília: Anvisa, 2004. v.1, p.52.

CARVAlho, J. C. T.; GOSMANN, G.; SCHENKEL, E. P. Compostos fenólicos simples e heterosídeos. In: SIMÕES, C. M. O; SCHENKEL, E. P.; GOSMANN, G.; MELLO, J. C. P.; MENTZ, L. A.; PETROVICK, P. R., (Orgs.). Farmacognosia: da planta ao medicamento. 5. ed. Porto Alegre/Florianópolis: Editora da UFRGS/editora da UFSC, 2003. p.1102.

ELMASTAS, M.; GÜLÇIN, I.; ISILDAK, Ö.; KÜFREVIOGLU, Ö. I.; IBAOGLU, K.; ABOUL-EINEN, H. Y. Radical scavenging activity and antioxidant capacity of bay leaf extracts. J. Iran Chem. Soc., v.3, n.3, p.258-266, 2006.
RAMALHO, V. C.; JORGE, N. Antioxidantes utilizados em óleos, gorduras e alimentos gordurosos. Quim. Nova, v.29, n.4, p. 755-760, 2006.

SILVA, E. C.; SOARES, I. C. Tecnologia de emulsões. Cosmet. Toilet. (Ed. port.). São Paulo, v.8, n. 5, p.38-45, 1996.

ZANIN, S. M. W.; MIGUEL, M. D.; CHIMELLI, M.; DALMAZ, A C. Parâmetros físicos no estudo da estabilidade das emulsões. Vis. Acad., v.2, n.2, p.47-58, 2001.

ZUNINO, G. P. A Farmácia Magistral no contexto da saúde. Pharm. Bras., v. 10, n.59-60, p.44-48, 2007.

Recebido para publicação em 09 de abril de 2008. Aceito para publicação em 07 de agosto de 2008. 